\title{
EVALUACIÓN DE LA PRECISIÓN DIAGNÓSTICA DE LA PUNCIÓN ASPIRACIÓN CON AGUJA FINA EN PACIENTES CON NÓDULO TIROIDEO
}

\author{
Hardenson Rodríguez González ${ }^{1}$ \\ Rafael Pava Marín² \\ Luisa Fernanda Castaño Herrera ${ }^{3}$ \\ Laura Vanesa Valencia García ${ }^{4}$ \\ Alex Pava Ripoll ${ }^{5}$
}

\section{RESUMEN}

Introducción: La punción aspiración con aguja fina ha desempeñado un papel esencial en la evaluación del paciente con nódulo tiroideo, siendo un método rápido, mínimamente invasivo y de bajo costo. El objetivo de este estudio fue evaluar la precisión diagnóstica de la punción aspiración con aguja fina en lesiones tiroideas, contrastando sus resultados con los hallazgos histopatológicos obtenidos en la pieza quirúrgica. Materiales y métodos: Se realizó un análisis retrospectivo utilizando los sistemas de registro informático del Instituto Caldense de Patología en Manizales - Colombia. Se analizaron los casos estudiados por punción aspiración con aguja fina de tiroides entre enero del 2012 a febrero del 2014. El estudio incluyó 1220 casos, de los cuales a 111 se les realizó tiroidectomía, los resultados se correlacionaron y se determinó sensibilidad, especificidad, valor predictivo positivo, valor predictivo negativo y precisión diagnóstica. Resultados: De los 111 casos, 5 se excluyeron por ser muestras insatisfactorias o atipias de significado indeterminado y quedaron 106 casos adecuados para la evaluación. La correlación citológica e histopatológica mostró una sensibilidad de $86,1 \%$, especificidad de $88,2 \%$, valor predictivo positivo de $93,9 \%$, valor predictivo negativo de $75 \%$ y una precisión diagnóstica de $86,7 \%$. Discusión: La punción aspiración con aguja fina de tiroides es una prueba sensible, específica y con una buena precisión diagnóstica. Los datos obtenidos son comparables con los hallados en estudios previos similares.

Palabras clave: nódulo tiroideo, tiroides, sensibilidad, especificidad.

\section{EVALUATION OF THE DIAGNOSTIC PRECISION OF FINE NEEDLE PUNCTURE ASPIRATION IN PATIENTS WITH THYROID NODULE}

\begin{abstract}
Introduction. The fine needle aspiration has played an essential role evaluating the patient with a thyroid nodule, is a rapid method, minimally invasive, with high diagnostic accuracy and low cost. The objective of this study evaluated the diagnostic accuracy of fine needle aspiration in thyroid lesions contrasting their results with histopathological findings obtained in the surgical specimen. Materials and methods:
\end{abstract}

\footnotetext{
${ }^{1}$ Estudiante de Medicina Universidad de Caldas, Manizales, Colombia. E-mail: hardenson.521022600@ucaldas.edu.co 2 Médico patólogo, docente asociado, Departamento de Ciencias Básicas, Facultad de Ciencias para la Salud, Universidad de Caldas, Manizales, Colombia. E-mail: Rafael.pava@ucaldas.edu.co

${ }_{3}^{3}$ Médico patólogo, docente asociado, Departamento de Ciencias Básicas, Facultad de Ciencias para la Salud, Universidad de Caldas, Manizales, Colombia. E-mail: Rafael.pava@ucaldas.edu.co

${ }^{4}$ Estudiante de Medicina Universidad de Caldas, Manizales, Colombia. E-mail: Laura.521020875@ucaldas.edu.co ${ }_{5}^{5}$ Médico patólogo, docente asistente Departamento de Ciencias Básicas, Facultad de Ciencias para la Salud, Universidad de Caldas y Docente de Universidad de Manizales, Manizales, Colombia. E-mail: Alex.pava@ucaldas.edu.co
} 
A retrospective analysis was performed using the informatics registry of Instituto Caldense de Patología in Manizales-Colombia. Were analyzed the cases of thyroid aspiration with fine needle studied from January 2012 to February 2014. The study included 1220 cases, of which 111 had a thyroidectomy, the results were correlated and sensibility, specificity, positive predictive value, negative predictive value and diagnostic accuracy were determined. Results: Of the 111 cases, 5 were excluded because the sample was unsatisfactory or because showed atypia of undetermined meaning, remaining 106 cases were evaluated. The cytological

\section{INTRODUCCIÓN}

Los nódulos palpables de la glándula tiroides son muy comunes; afectan aproximadamente del 4 al 7\% de la población general (1). La mayoría son asintomáticos, son cuatro veces más frecuentes en mujeres que en hombres y la incidencia aumenta con la edad. Se han considerado como antecedentes de importancia la exposición a radiación ionizante y la dieta que contenga material bociogénico (2). La mayoría de los nódulos tiroideos son benignos y solo entre un 5 y un $10 \%$ resultan ser lesiones malignas (3).

Las neoplasias tiroideas tienen un amplio espectro de presentaciones que van desde lesiones foliculares benignas hasta el agresivo cáncer anaplásico (4). La tiroidectomía total se considera como el procedimiento de elección para los diferentes tipos de cáncer de tiroides (5).

La punción aspiración con aguja fina (PAAF) ha desempeñado un papel esencial en la evaluación del paciente con un nódulo tiroideo, siendo un método rápido, mínimamente invasivo y de bajo costo, ya que reduce cirugías innecesarias para los pacientes con nódulos tiroideos benignos y clasifica apropiadamente a los pacientes con nódulos malignos para la intervención quirúrgica oportuna $(6,7)$. and histopathological correlation showed a sensibility of $86.1 \%$, specificity of $88.2 \%$, positive predictive value of $93.9 \%$, negative predictive value of $75 \%$, and a diagnostic accuracy of $86.7 \%$. Discussion: Thyroid fine needle aspiration is a sensitive, specific test with good diagnostic accuracy. Data obtained are comparable to those found in similar previous studies. When an inadequate or unsatisfactory sample is found, the biopsy must be repeated.

Key words: thyroide nodule, thyroid gland, sensitivity, specificity

A pesar de su gran valor, la PAAF puede presentar algunos problemas dentro de los cuales se incluyen: punciones inadecuadas, la incapacidad de distinguir de forma fiable el adenoma folicular del carcinoma folicular y la posibilidad de falsos negativos y falsos positivos (8-12). Tomada adecuadamente e interpretada por un patólogo experto, la PAAF debería tener un porcentaje de falsos negativos inferior al 5\% (13).

El objetivo de este estudio fue evaluar la precisión diagnóstica de la PAAF en nódulos tiroideos y contrastar sus resultados con los hallazgos histopatológicos obtenidos en la pieza quirúrgica.

\section{MATERIALES Y MÉTODOS}

Se realizó un análisis retrospectivo utilizando los sistemas de registro informático del Instituto Caldense de Patología (ICP) en Manizales Colombia. Se analizaron los casos estudiados por PAAF de tiroides entre enero del 2012 a febrero del 2014. El estudio incluyó 1220 pacientes con nódulos tiroideos a los que se les realizó PAAF con la técnica estándar descrita en la literatura (14), utilizando agujas calibre 23 - 25 conectadas a jeringas de $10 \mathrm{ml}$. El material fue fijado con alcohol al 96\% y se tiñó con hematoxilina-eosina.

Los resultados de las PAAF de cada paciente fueron clasificadas por los patólogos del ICP 
en 6 categorías de acuerdo al sistema Bethesda (15), así: I: no diagnóstica o insatisfactoria, II: benigno, III: atipia de significado indeterminado o lesión folicular de significado indeterminado, IV: neoplasia folicular o sospechoso de neoplasia folicular, V: sospechoso de malignidad, VI: maligno.

Para cada paciente se recogieron los datos demográficos de edad y sexo; de los 1220 pacientes con PAAF a 111 se les realizó posteriormente tiroidectomía. La indicación quirúrgica se estableció a criterio de los médicos encargados y es independiente de los objetivos de este estudio, además se recolectó la información del reporte histopatológico post quirúrgico, el cual fue comparado con el diagnóstico previo realizado con la PAAF. Los casos que resultaron ser positivos para malignidad mediante la $\mathrm{PAAF}$, al igual que en la histopatología, fueron etiquetados como verdaderos positivos (VP), los falsos positivos (FP) fueron los diagnosticados como positivos para malignidad en la PAAF pero benignos en la histopatología, verdaderos negativos (VN) fueron los negativos para malignidad tanto en la PAAF como en la histopatología, falsos negativos (FN) fueron negativos para malignidad en la PAAF pero positivos para malignidad en la histopatología. Finalmente, se estableció la correlación entre los resultados de la PAAF y la histopatología por medio de una matriz de decisiones o tabla de $2 \times 2$ para determinar sensibilidad, especificidad, valor predictivo positivo, valor predictivo negativo y precisión diagnóstica, las fórmulas estadísticas para determinar estos resultados se pueden observar en la tabla 3 (16). Los casos de categoría I (no satisfactoria o indeterminado) y categoría III (atipia de significado indeterminado o lesión folicular de significado indeterminado), fueron excluidos del cálculo.

\section{RESULTADOS}

Se identificaron en los registros del Instituto Caldense de Patología 1220 casos de PAAF de tiroides en el periodo de enero del 2012 a febrero del 2014. La distribución por sexos fue de 1128 mujeres $(92,5 \%)$ y 92 varones $(7,5 \%)$. El rango de edad de la población estudiada fue de 9 a 100 años, con una edad media de 56,67.

Del total de pacientes con PAAF, 103 (8,4\%) fueron categoría I: no diagnóstica/insatisfactoria según la clasificación Bethesda, 890 (73\%) categoría II: benigna, 41 (3,4\%) categoría III: atipia de significado indeterminado o lesión folicular de significado indeterminado, 59 (4,8\%) categoría IV: neoplasia folicular o sospechoso de neoplasia folicular, $38(3,1 \%)$ categoría $\mathrm{V}$ : sospechoso de malignidad y 89 (7,3\%) como categoría VI: maligno (Tabla 1).

Tabla 1 - Distribución de diagnósticos en 1220 PAAF.

\begin{tabular}{cc}
\hline Categorías Bethesda & Número de casos \\
\hline I & 103 \\
II & 890 \\
III & 41 \\
IV & 59 \\
V & 38 \\
VI & 89 \\
Total & 1220 \\
\hline
\end{tabular}

I: No diagnóstica o Insatisfactoria, II: Benigno, III: Atipia de Significado Indeterminado o Lesión Folicular de Significado Indeterminado, IV: Neoplasia Folicular o Sospechoso de Neoplasia Folicular, V: Sospechoso de Malignidad, VI: Maligno. 
Hardenson Rodríguez González et al.

De los 1220 casos, a 111 se les realizó tiroidectomía posteriormente, cinco fueron excluidos dado que tenían categoría I y III de Bethesda quedando 106 casos disponibles para el análisis. De éstos, se realizó el diagnóstico histopatológico de bocio en 24 casos $(22,6 \%)$, tiroiditis de Hashimoto en tres casos $(2,8 \%)$, tiroiditis linfocitaria en un caso $(0,9 \%)$, adenoma folicular en nueve casos $(8,4 \%)$, neoplasia folicular en un caso $(0,9 \%)$, carcinoma folicular en dos casos (1,8\%) y carcinoma papilar en 66 casos $(62,2 \%)$ (Tabla2).

Tabla 2 - Distribución de diagnósticos histopatológicos en 106 casos

\begin{tabular}{lc}
\hline Resultado Histopatológico & Número de casos \\
\hline Bocio & 24 \\
Tiroiditis de Hashimoto & 3 \\
Tiroiditis Linfocitaria & 1 \\
Adenoma Folicular & 9 \\
Neoplasia Folicular & 1 \\
Carcinoma Folicular & 1 \\
Carcinoma Papilar & 66 \\
Total & 106 \\
\hline
\end{tabular}

Con base en la información recolectada se obtuvieron los siguientes resultados para la PAAF de tiroides: sensibilidad de $86,1 \%$ (62/72), especificidad de $88,2 \%(30 / 34)$, valor predictivo positivo de $93,9 \%(62 / 66)$, valor predictivo negativo de $75 \%$ (30/40). La precisión diagnóstica calculada para la PAAF fue de $86,7 \%$ (92/106) (Tabla 3).

Tabla 3 - Matriz de decisiones o tabla de 2x2(resultados de la PAAF y resultados histopatológicos)

\begin{tabular}{llll}
\hline PAAF & $\begin{array}{l}\text { Histopatología } \\
\text { Maligno }\end{array}$ & No maligno & Total \\
\hline Positiva $^{*}$ & $62(\mathrm{VP})$ & $4(\mathrm{FP})$ & $66(\mathrm{E})$ \\
Negativa $^{\dagger}$ & $10(\mathrm{FN})$ & $30(\mathrm{VN})$ & $40(\mathrm{~F})$ \\
Total & $72(\mathrm{G})$ & $34(\mathrm{H})$ & $106(\mathrm{~T})$ \\
$\mathrm{S}=\mathrm{VP} / \mathrm{G}$ & $\mathrm{VPP}=\mathrm{VP} / \mathrm{E}$ & & $\mathrm{AD}=\mathrm{VP}+\mathrm{VN} / \mathrm{T}$ \\
$\mathrm{E}=\mathrm{VN} / \mathrm{H}$ & $\mathrm{VPN}=\mathrm{VN} / \mathrm{F}$ & & \\
\hline
\end{tabular}

*Positiva para malignidad; ${ }^{+}$negativa para malignidad; VP: verdadero positivo; FP: falso positivo; FN: falso negativo; VN: verdadero negativo; S: sensibilidad; E: especificidad; VPP: valor predictivo positivo; VPN: valor predictivo negativo; PD: precisión diagnóstica. 


\section{DISCUSIÓN}

La PAAF se considera el método de elección en el estudio de pacientes con nódulo tiroideo debido a que contribuye a la diferenciación de nódulos benignos y malignos; con el uso de esta herramienta diagnóstica se deberían determinar cuáles pacientes deben ser tratados quirúrgicamente(27). Su uso adecuado disminuye el porcentaje de personas intervenidas con lesiones no malignas del $40 \%$ al $3 \%$ (28). Exponer a todos los pacientes a cirugía es impráctico, ya que la mayoría presentan nódulos benignos como lo demuestra nuestro estudio, teniendo en cuenta que la intervención trae consigo riesgos considerables.

Para que una PAAF se considere de utilidad clínica debe contener una muestra satisfactoria; para lograrlo, el material tomado debe ser representativo de la lesión, con una cantidad adecuada y una buena preparación citológica. Entre el 2 al 21\% (29) de los casos en estudios previos han correspondido a la categoría I de Bethesda (no diagnóstica o insatisfactoria), siendo ésta la única indicación de repetir la PAAF, dado que ya está demostrado que someter todos los casos a aspiraciones repetidas no mejora el rendimiento diagnóstico (11). En este estudio se obtuvieron $103(8,4 \%)$ muestras insatisfactorias o no diagnósticas, resultado que es comparable con los obtenidos en otros estudios como ya se hizo mención.

Se obtuvo para la PAAF una sensibilidad de $86,1 \%$, una especificidad de $88,2 \%$ y una precisión diagnóstica de $86,7 \%$, resultados que comparados con estudios previamente realizados son similares y están en los rangos establecidos como es una sensibilidad que va desde 54 - 99,8\% (19,23), especificidad de 35 $100 \%(20,24)$ y precisión diagnóstica de $67,2-97 \%$ $(17,19)$. Los resultados obtenidos en este estudio son comparados con otros 15 estudios desde el año 2000 en la tabla 4. Un factor que influye en la variabilidad de estos resultados es tomar para el cálculo de los índices, conjuntamente a los positivos para malignidad, a los sospechosos de malignidad y a los indeterminados. Lo anterior justificado en el hecho de que es imposible distinguir únicamente por medio de la PAAF, a las neoplasias foliculares malignas (carcinomas) y las benignas (adenomas), ya que para identificar a las primeras se debe demostrar invasión vascular o capsular en la histopatología de la muestra quirúrgica $(24,30)$. En este estudio no se tuvieron en cuenta para el cálculo de los índices, las muestras con categoría III de Bethesda que equivalen a atipia de significado indeterminado o lesión folicular de significado indeterminado, pero sí se consideraron las de categoría IV (neoplasia folicular o sospechoso de neoplasia folicular). Al obtener un resultado de categoría IV de Bethesda, se recomienda tener en cuenta información clínica de factores que podrían aumentar el riesgo de malignidad, como son pacientes con más de 40 años de edad, con nódulos de más de $3 \mathrm{~cm}$ de tamaño y de sexo masculino (9).

Se clasificaron como categoría $V$ o sospechosos de malignidad 38 casos $(3,1 \%)$ de los 1220 con PAAF, de los cuales se obtuvo registro de tiroidectomía en diez casos, encontrando nueve casos de histopatología con carcinoma papilar y 1 con bocio. Este hallazgo apoya el hecho de intervenir quirúrgicamente los nódulos tiroideos considerados sospechosos de malignidad por la PAAF sin necesidad de repetirla (31).

Se encontró que la PAAF de tiroides es una prueba sensible, específica y con una buena precisión diagnóstica. Los datos obtenidos son comparables con los hallados en estudios previos similares, lo que demuestra que en nuestro medio la toma y análisis de las PAAF son apropiados (Tabla 4) y evidencia que es un método valioso, fiable y adecuado para evaluar pacientes con nódulos tiroideos. El hallazgo de muestra inadecuada o no satisfactoria es indicación de repetir la PAAF. Debido a las limitaciones ya discutidas, es fundamental determinar la conducta de los pacientes con nódulo tiroideo, no solo basados en el 
Hardenson Rodríguez González et al.

resultado de la PAFF, sino también teniendo en cuenta otros indicadores como son la historia clínica, el examen físico, los exámenes de laboratorio e imágenes diagnósticas. Dado el alto porcentaje de malignidad, es recomendable intervenir quirúrgicamente los casos en los que se encuentre PAAF con diagnóstico en la categoría $\mathrm{V}$ (sospechoso de malignidad) o categoría VI (maligna) del sistema Bethesda.

Tabla 4 - Comparación de resultados de este estudio con otros reportados a partir del año 2001

\begin{tabular}{|c|c|c|c|c|c|c|}
\hline Autores & Año & $\mathrm{S}(\%)$ & E (\%) & VPP & VPN & PD (\%) \\
\hline Amrikachi et al. ${ }^{2}$ & 2001 & 93,0 & 96,0 & - & - & - \\
\hline Morgan et al. ${ }^{17}$ & 2003 & 55,0 & 73,7 & 70,0 & 67,4 & 67,2 \\
\hline Jogal et al. ${ }^{18}$ & 2005 & 88,4 & 99,1 & - & - & 78,1 \\
\hline Carrera et al. ${ }^{11}$ & 2005 & 76,0 & 83,0 & 48,0 & 95,0 & 82,0 \\
\hline Cai et al. ${ }^{19}$ & 2006 & 83,3 & 98,0 & 71,4 & 98,4 & 97,0 \\
\hline Cheung $^{20}$ & 2007 & 54,0 & 100 & 100 & 75,0 & - \\
\hline Tseng et al. ${ }^{10}$ & 2008 & 81,0 & 98,7 & 94,4 & 95,0 & 94,9 \\
\hline Rubio et al..$^{21}$ & 2009 & 74,4 & 73,1 & 32,9 & 94,1 & - \\
\hline Khan et al. ${ }^{12}$ & 2010 & 75,0 & 96,0 & 81,0 & 95,0 & 93,0 \\
\hline Swamy ${ }^{22}$ & 2011 & 75,0 & 95,8 & 81,8 & 93,8 & 96,6 \\
\hline Sinna et al. ${ }^{23}$ & 2012 & 92,8 & 94,2 & 94,9 & 91,8 & 93,6 \\
\hline Chala et al..$^{24}$ & 2013 & 81,4 & 89,4 & 87,7 & 84,1 & - \\
\hline Prades et al. ${ }^{25}$ & 2013 & 99,2 & 35,7 & 93,7 & 83,3 & 84,2 \\
\hline Naz et al. ${ }^{26}$ & 2014 & 66,3 & 85,1 & 56,3 & 88,9 & 80,3 \\
\hline Este estudio & 2016 & 86,1 & 88,2 & 93,9 & 75,0 & 86,7 \\
\hline
\end{tabular}

$\mathrm{S}=$ sensibilidad; $\mathrm{E}=$ especificidad $\mathrm{VPP}=$ valor predictivo positivo; $\mathrm{VPN}=$ valor predictivo negativo; $\mathrm{PD}=$ precisión diagnóstica; - = datos desconocidos 


\section{REFERENCIAS BIBLIOGRÁFICAS}

1. Welker MJ, Orliv D. Thyroid nodules. Am Fam Physician 2003; 67:559-66. Disponible en: https:// www.ncbi.nlm.nih.gov/pubmed/12588078

2. Amrikachi M, Ramzy I, Rubenfeld S, Wheeler TM. Accuracy of fine-needle aspiration of thyroid. Arch Pathol Lab Med 2001; 125:484-8.DOI: 10.1043/0003-9985(2001)125<0484:AOFNAO>2.0.CO;2

3. Aysan $E$, Kiran $T$, Idiz UO, Guler $B$, Akbulut $H$, Kunduz $E$, et al. The diagnostic ability of core needle biopsy in nodular thyroid disease. Ann R Coll Surg Eng/ 2016; 00:1-4. DOI:10.1308/rcsann.2016.0331 [In press]

4. Abdul-Jaber HB, Lynn J. The surgical management of thyroid cancer. Nucl Med Commun 2004; 25:869-72. Disponible en: http://journals.Iww.com/nuclearmedicinecomm/Citation/2004/09000/ The_surgical_management_of_thyroid_cancer.2.aspx

5. Mishra A, Mishra SK. Total thyroidectomy for differentiated thyroid cancer: primary compared with completion thyroidectomy. Eur J Surg 2002; 168:283-7. DOI:10.1002/ejs.47

6. Yang J, Schnadig V, Logrono R, Wasserman PG. Fine-needle aspiration of thyroid nodules: a study of 4703 patients with histologic and clinical correlations. Cancer Cytopathology 2007; 111:306-15. DOI: $10.1002 /$ cncr.22955

7. Mateša N, Tabain I, Dabelić N, Petric V, Kusić Z. Diagnostic relevance of fine-needle aspiration cytology for follicular lesions of the thyroid: retrospective study. Croat Med J 2002; 43:606-9. Disponible en: https://www.ncbi.nlm.nih.gov/pubmed/12402405

8. Bajaj Y, De M, Thompson A. Fine needle aspiration cytology in diagnosis and management of thyroid disease. J Laryngol Otol 2006; 120:467DOI: 10.1017/S0022215106000703

9. Baloch ZW, Fleisher S, LiVolsi VA, Gupta PK. Diagnosis of "follicular neoplasm": a gray zone in thyroid fine-needle aspiration cytology. Diagn Cytopathol 2002; 26:41-4.DOI: 10.1002/dc.10043

10. Tseng CE, Wei CK, Kuo CS, Yan ST, Chen PF, Lien WC, et al. Fine needle aspiration cytology of thyroid nodules: evaluation of diagnostic accuracy. Tzu Chi Medical Journal 2008; 20:296-303. DOI:10.1016/ S1016-3190(08)60054-3

11. Carrera CB, García-Díaz JD, Villaizán EM, Martínez-Onsurbe P, Torres NP, Vallejo PS. Eficacia diagnóstica de la punción-aspiración con aguja fina en pacientes con patología nodular tiroidea. Análisis de 510 casos. Revista Clínica Española 2005; 205:374-378. DOI: 10.1157/13078248

12. Khan I, Naz S, Akhter ZM, Aziz N. Diagnostic accuracy of fine needle aspiration of thyroid nodule verses biopsy in thyroid lesions. J Ayub Med Coll Abbottabad 2010; 22:179-181. Disponible en: https://www.ncbi.nlm.nih.gov/pubmed/22455292\#

13. Gharib H. Fine-needle aspiration biopsy of thyroid nodules: advantages, limitations, and effect. Mayo Clin Proceedings 1994; 69:44-9. DOI: 10.1016/S0025-6196(12)61611-5

14. Orell SR, Sterrett GF, Walters MN, Whitaker D. Head and neck. In: Orell SR, Sterrett GF, Walters MN, Whitaker D. (Eds). Manual and Atlas of fine needle aspiration cytology. 3a Ed. Londres: Elsevier Science Limited; 1999. p. 110-44.

15. Ali, S.Z., \& Cibas, E.S. The Bethesda system for reporting thyroid cytopathology: definitions, criteria and explanatory notes. Springer; 2010.

16. Nurismah MI, Sharifah NA, Usama AE, Rohaizak M, Naqiyah I, Jasmi A. Fine needle aspiration (FNA) cytology of thyroid: a cytohistopathological study of 361 cases in hospital university Kebangsaan Malaysia. Medicine \& Health 2007; 2:58-65. Disponible en: http://www.medicineandhealthukm.com/ fulltext/385

17. Morgan JL, Serpell JW, Cheng MS. Fine-needle aspiration cytology of thyroid nodules: how useful is it? ANZ Journal of Surgery 2003; 73:480-3. DOI: 10.1046/j.1445-1433.2003.02670.x

18. Jogai $S$, Al-Jassar A, Temmim L, Dey $P$, Adesina AO, Amanguno HG. Fine needle aspiration cytology 
Hardenson Rodríguez González et al.

of the thyroid: a cytohistologic study with evaluation of discordant cases. Acta Cytol 2005; 49:483-8. DOI: $10.1159 / 000326192$

19. Cai XJ, Valiyaparambath N, Nixon P, Waghorn A, Giles T, Helliwell T. Ultrasound-guided fine needle aspiration cytology in the diagnosis and management of thyroid nodules. Cytopathology 2006; 17:251-6. DOI: $10.1111 /$ j.1365-2303.2006.00397.x

20. Cheung YS, Poon CM, Mak SM, Suen MW, Leong HT. Fine-needle aspiration cytology of thyroid nodules-how well are we doing? Hong Kong medical journal 2007; 13:12-15. Disponible en: http:// www.hkmj.org/abstracts/v13n1/12.htm

21. Rubio V, Tamarit JM, Baviera N, Fernández S, Estrems P, Seijas T, López C, Dalmau J. (2009). Evaluación metodológica para detección de malignidad en patología quirúrgica tiroidea. Acta Otorrinolaringológica Española 2009; 60:390-395.DOI: 10.1016/j.otorri.2009.07.004

22. Swamy GG, Madhuravani S, Swamy GM. Fine needle aspiration cytology - a reliable diagnostic tool in the diagnosis of thyroid gland enlargements. Nepal Medical College Journal 2011; 13:289-292. Disponible en: https://www.ncbi.nlm.nih.gov/pubmed/23016483

23. Sinna EA, Ezzat N. Diagnostic accuracy of fine needle aspiration cytology in thyroid lesions. Journal of the Egyptian National Cancer Institute 2012; 24:63-70. DOI: 10.1016/j.jnci.2012.01.001

24. Chala AI, Pava R, Franco HI, Álvarez A, Franco A. Criterios ecográficos diagnósticos de neoplasia maligna en el nódulo tiroideo: correlación con la punción por aspiración con aguja fina y la anatomía patológica. Rev Colombiana Cir 2013; 28:15-23. Disponible en: http://www.scielo.org.co/pdf/rcci/ v28n1/v28n1a2.pdf

25. Prades JM, Querat C, Dumollard JM, Richard C, Gavid M, Timoshenko AA, Peoc'h M, Martin C. Thyroid nodule surgery: Predictive diagnostic value of fine-needle aspiration cytology and frozen section. European annals of otorhinolaryngology, head and neck diseases 2013; 130:195-199.DOI: 10.1016/j. anorl.2012.12.005

26. Naz S, Hashmi AA, Faridi N, Edhi MM, Kamal A, Khan M. Diagnostic accuracy of Bethesda system for reporting thyroid cytopathology: an institutional perspective. International Archives of Medicine 2014; 7:46. DOI: $10.1186 / 1755-7682-7-46$

27. Lee TI, Yang HJ, Lin SY, et al. The accuracy of fine-needle aspiration biopsy and frozen section in patients with thyroid cancer. Thyroid 2002; 12:619-26. DOI:10.1089/105072502320288492

28. Ahmeti I, Simonovska L, Krstevska B, Ristevska N. Fine Needle Aspiration in Thyroid Nodules - One Year Experience. OA Maced J Med Sci 2015; 3(2):307-309.DOI:10.3889/oamjms.2015.064

29. Ravetto C, Colombo L, Dottorini ME. Usefulness of fine-needle aspiration in the diagnosis of thyroid carcinoma: a retrospective study in 37,895 patients. Cancer Cytopathology 2000; 90:357-63. DOI: 10.1002/1097-0142(20001225)90:6<357::AID-CNCR6>3.0.CO;2-4

30. Werga $P$, Wallin G, Skoog L, Hamberger B. Expanding role of fine-needle aspiration cytology in thyroid diagnosis and management. World J Surg 2000; 24:907-12. Disponible en: https://www.ncbi.nlm. nih.gov/pubmed/10865034

31. Greaves TS, Olvera M, Florentine BD, Raza AS, Cobb CJ, Tsao-Wei DD, et al. Follicular lesions of thyroid. Cancer Cytopathology 2000; 90:335-41. DOI: 10.1002/1097-0142(20001225)90:6<335: :AIDCNCR3 $>3.0 . \mathrm{CO} ; 2-\mathrm{L}$ 